\title{
INTRODUCTIONS DE POISSONS D'EAU DOUCE ET D'ÉCREVISSES EN CAMARGUE : HISTORIQUE, ORIGINES ET MODIFICATIONS DES PEUPLEMENTS.
}

\author{
E. ROSECCHI, G. POIZAT et A.J. CRIVELLI.
}

Station Biologique de la Tour du Valat, Le Sambuc, 13200 Arles, France.

\section{RÉSUMÉ}

Treize espèces de poissons dulçaquicoles exotiques sont actuellement recensées en Camargue, dans le delta du Rhône. Parmi elles, onze sont naturalisées. Deux écrevisses américaines ont également été recensées. Pour illustrer les modifications intervenues dans les peuplements de poissons à la suite de ces introductions, nous prenons l'exemple d'un canal de drainage. Les impacts potentiels (compétition alimentaire, hybridation) sur les espèces autochtones sont identifiés et discutés. Nous avons tenté de caractériser chez Pseudorasbora parva les traits d'histoire de vie qui ont permis l'établissement rapide de cette espèce exotique dans le canal. Des suggestions sont émises en conclusion, pour une meilleure compréhension des interactions entre espèces introduites et faune autochtone.

\section{THE INTRODUCTION OF FRESHWATER FISH AND CRAYFISH SPECIES IN THE CAMARGUE : HISTORY, ORIGINS AND CHANGES IN ASSEMBLAGES.}

\begin{abstract}
Thirteen introduced freshwater fish species were recorded in the Camargue (Rhone Delta, Southern France). Eleven of them have established a population. Two American freshwater crayfishes were also introduced. The example of a drainage canal (studied between 1989 and 1995) was chosen to illustrate the changes in fish assemblages that occurred after those introductions. Depending on the year, the relative abundance (CPUE) of exotic species represented from $27 \%$ to $33 \%$ of the total catch. The potential impacts of those introductions (diet overlap, hybridization) on natural fish communities are identified and discussed. Life history traits of Pseudorasbora parva, an Asiatic species first recorded in 1993 in the canal, have been studied; some of them might explain the successful and fast establishment of this species in the Camargue. In conclusion, suggestions and recommendations are proposed for a better understanding of the mechanisms involved in the interactions between introduced species and native communities.
\end{abstract}

\section{INTRODUCTION}

Dans le monde, 291 espèces de poissons dulçaquicoles ont été introduites en dehors de leur zone de distribution originelle et le taux d'introduction ne cesse d'augmenter (WELCOMME, 1992). Pourtant, si les effets écologiques potentiels des introductions d'espèces terrestres sont connus depuis longtemps, ceux afférents aux poissons n'ont reçu l'attention des biologistes que depuis les années 60 (COURTENAY, 1989). Les conséquences de ces introductions sont toujours sujettes à controverse et font surtout l'objet de débats entre gestionnaires des pêcheries et conservateurs de la nature (CRIVELLI, 1995). 
La littérature sur l'impact des introductions d'espèces exotiques comprend de nombreux exemples de pertes dramatiques d'espèces autochtones. Cependant, les relations de cause à effet restent souvent hypothétiques, faute d'information sur le statut des espèces autochtones avant l'introduction et faute d'un suivi à long terme par la suite (TOWNSEND et WINTERBOURN, 1992 ; CRIVELLI, 1995). De plus, la perturbation des habitats parallèlement à I'introduction d'espèces exotiques rend difficile l'interprétation de tout changement intervenu dans les populations naturelles (ARTHINGTON, 1991 ; WINFIELD, 1992).

Dans les années 50, un inventaire ichtyologique de l'île de Camargue, delta du Rhône, avait permis de dénombrer 34 espèces dulçaquicoles et euryhalines, dont six introduites (LÉVÊQUE, 1957). Dans une étude réalisée entre 1977 et 1980 dans les eaux douces et saumâtres de Camargue, CRIVELLI (1981) recensait 39 espèces dont les mêmes six espèces exotiques et aucune autre. En 1995, le nombre d'espèces exotiques était de 13, pour 26 espèces autochtones.

Le but de notre travail est de dresser le bilan des introductions de poissons et macrocrustacés en Camargue, et de tenter de déterminer si ces espèces exotiques ont pu établir des populations. Nous avons étudié certains de leurs impacts sur les peuplements de poissons autochtones (chevauchement des niches alimentaires, hybridation) et tenté de caractériser chez Pseudorasbora les traits d'histoire de vie qui ont pu permettre le succès de cette espèce exotique. Enfin, nous émettrons quelques suggestions pour une meilleure compréhension des interactions entre espèces introduites et faunes autochtones.

\section{MATÉRIEL ET MÉTHODES}

Cette étude concerne la Camargue Est (à l'est du Vaccarès), ainsi que le Plan du Bourg (à l'est du Grand Rhône), à l'exception du cours du Rhône lui-même. Nos points d'échantillonnage ont couvert sur la période 1976-1995 des marais d'eau douce, des canaux de drainage et d'irrigation en Camargue, les marais et canaux du Vigueirat et de Sollac (Plan du Bourg), des lagunes saumâtres (étang du Tampan, étang du Vaccarès) (Fig. 1).

Un échantillonnage régulier des peuplements de poissons d'eau douce et de macroinvertébrés de Camargue fut mis en place dans le canal du Fumemorte (Fig. 1). Ce canal reçoit l'eau de drainage, principalement celle des rizières depuis 1950 , et coule par gravité vers le Vaccarès, étang saumâtre de 6700 ha. Le Vaccarès communique de façon intermittente avec la mer, via les étangs inférieurs, par des martellières situées au Grau de la Fourcade (Fig. 1). Le canal du Fumemorte a une profondeur moyenne de 0,5 à 1,5 $\mathrm{m}$. Sa salinité est restée comprise entre 0,16 et $3,09 \mathrm{~g} / \mathrm{l}$ pour la période $1988-1995$, avec une moyenne de $0,94 \mathrm{~g} / \mathrm{l}(\mathrm{n}=303$, écarttype 0,59).

L'échantillonnage dans le Fumemorte a été réalisé au moyen de filets trémails (diverses mailles) dès 1982 et de ganguis (maille $5 \mathrm{~mm}$ ) dès 1988. Pour une année d'échantillonnage, tout engin restait calé plusieurs jours par mois et était visité tous les jours. Pour chaque engin et pour chaque espèce, nous avons calculé les captures par unité d'effort (CPUE), en nombre d'individus par 24 heures de pêche, transformées en Ln (CPUE +1$)$. Les régimes alimentaires des espèces, caractérisés par les fréquences d'occurrence des principales catégories d'aliments dans 897 contenus stomacaux, ont été comparés par le coefficient de corrélation de Spearman et une analyse factorielle des correspondances. Le nombre de contenus par espèce est compris entre 28 et 143 , excepté pour la carpe hybride $(n=17)$ et le silure $(n=9)$.

Les traits d'histoire de vie de Pseudorasbora parva ont été étudiés sur 318 individus pêchés dans le canal du Fumemorte en 1995, traités selon les méthodes décrites dans ROSECCHI et al. (1993). Ces traits ont été comparés avec ceux observés dans le lac Prespa (ROSECCHI et al., 1993).

L'étude morphométrique des carpes du Fumemorte a pris en compte 13 variables morphométriques (dont la mesure a été divisée par la longueur standard du poisson) : longueur 


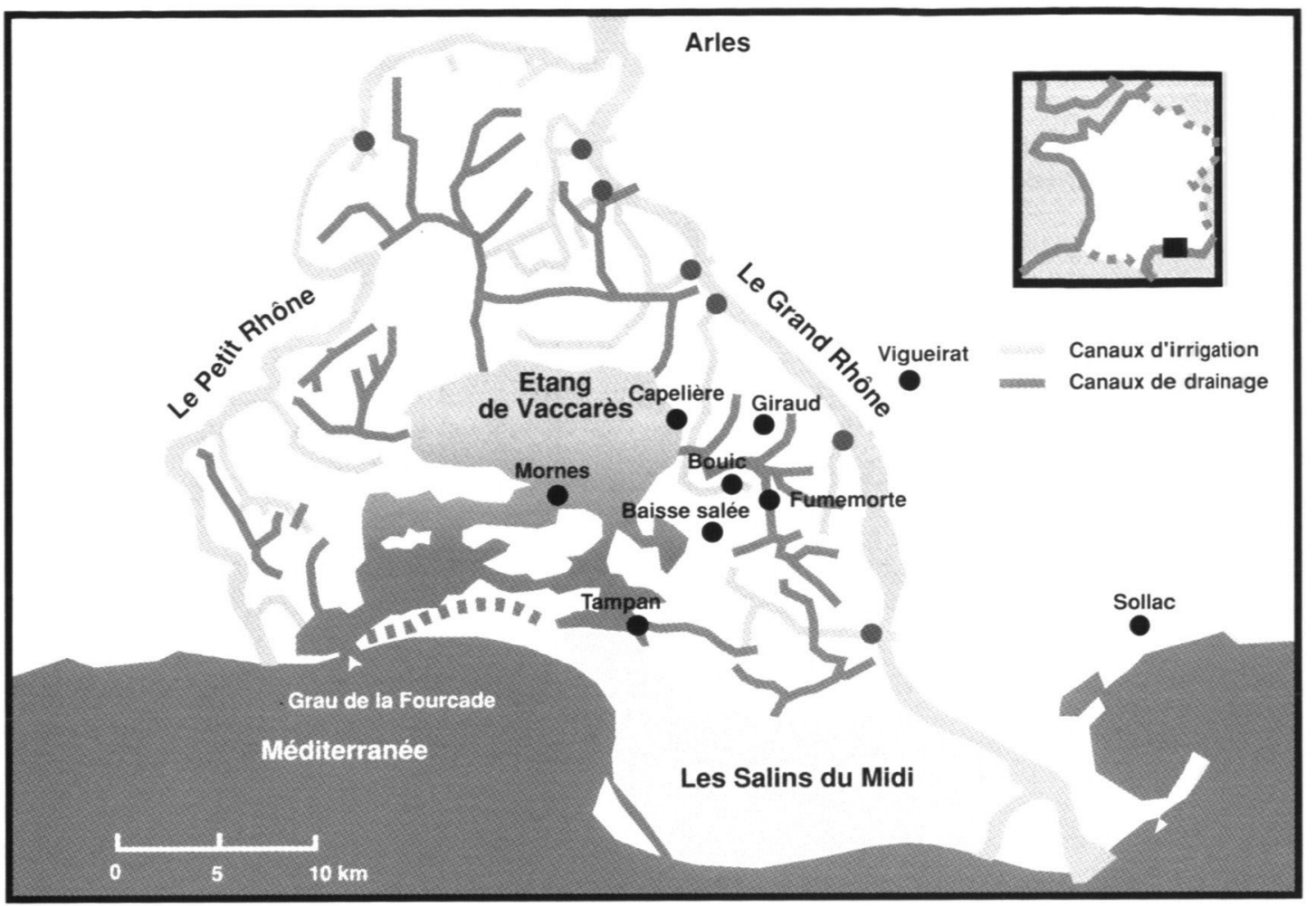

Figure 1

Sites d'échantillonnage en Camargue (delta du Rhône).

Figure 1

Sampling sites in Camargue (Rhone delta).

de la tête, distances anté-dorsale et post-dorsale, hauteurs maximale et minimale du corps, longueur de la base et hauteur des nageoires dorsale et anale, longueur des nageoires pectorales et pelviennes, diamètre de l'oeil, distances anté-ventrale et anté-anale. 5 variables méristiques ont été considérées : nombre d'écailles le long de la ligne latérale, nombre de rayons aux nageoires dorsale, anale, pelviennes et pectorales. Le rapport entre le diamètre de l'oeil et la longueur de la tête a également été pris en compte. Une analyse discriminante (CHESSEL et DOLEDEC, 1994) a été réalisée pour obtenir un axe discriminant les carpes sauvages et de pisciculture. Les individus supposés hybrides y ont ensuite été projetés en individus supplémentaires.

\section{RÉSULTATS}

\section{Historique et origines des introductions}

La liste des espèces exotiques pour la Camargue (Tableau I) est la synthèse de vingt années d'échantillonnage (1976-1995). Pour certaines espèces, cette étude constitue pour la Camargue la première mention dans la littérature ; c'est le cas par exemple du Black-bass Micropterus salmoïdes, déjà signalé dans le Plan du Bourg en 1952, mais uniquement dans des documents non publiés.

Etant donné les dates d'apparition dans les captures, nous avons la certitude que l'introduction de certaines espèces dans le milieu est très récente (Tableau I). Plus de $40 \%$ des espèces introduites le sont à la suite d'incidents survenus dans des piscicultures voisines. Les marais de pisciculture sont régulièrement vidangés et les canaux d'évacuation communiquent indirectement avec le canal du Fumemorte. L'écrevisse de Louisiane, déjà signalée dans le Gard (ROQUEPLO et al., 1995), pourrait avoir colonisé naturellement la Camargue. 
Tableau I

Liste des espèces de poissons et macro-crustacés dulçaquicoles exotiques capturëes en Camargue (delta du Rhône).

\section{Table I}

List of exotic freshwater fish and macro-crustaceans species collected in Camargue (Rhone delta).

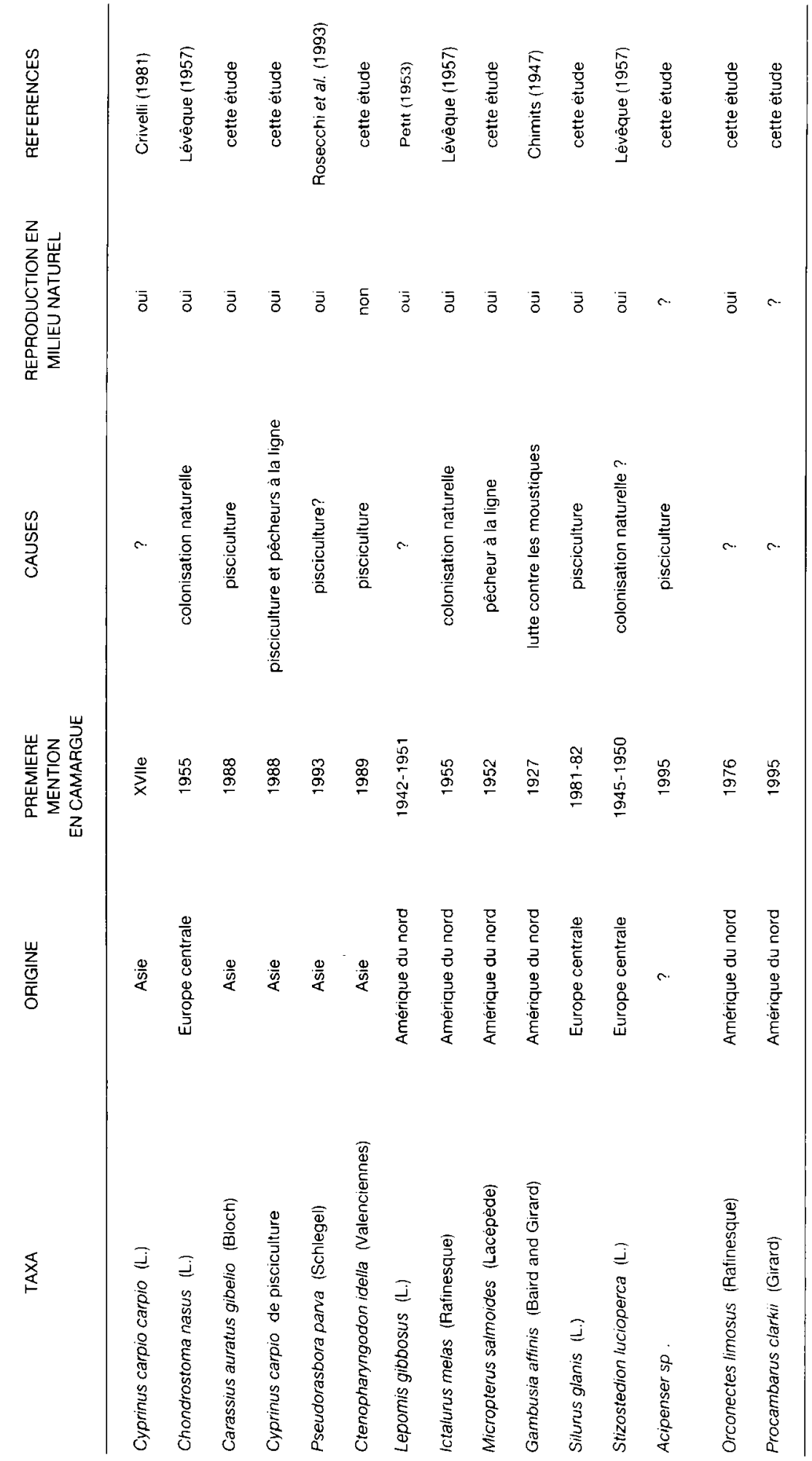


Toutes les espèces introduites se reproduisent en Camargue, à l'exception de trois d'entre elles (Tableau I). La carpe amour Ctenopharyngodon idella a des exigences très strictes pour sa reproduction qui ne sont pas réunies dans cet habitat et, de plus, la plupart des individus d'élevage sont triploïdes, ce qui les rend fonctionnellement stériles (BAIN, 1993). N'ayant capturé qu'un seul individu d'écrevisse de Louisiane, Procambarus clarkii, et trois individus d'esturgeon, Acipenser sp., nous ne savons pas encore si ces espèces sont naturalisées. et 1995

Modifications du peuplement de poissons d'un canal de drainage entre 1989

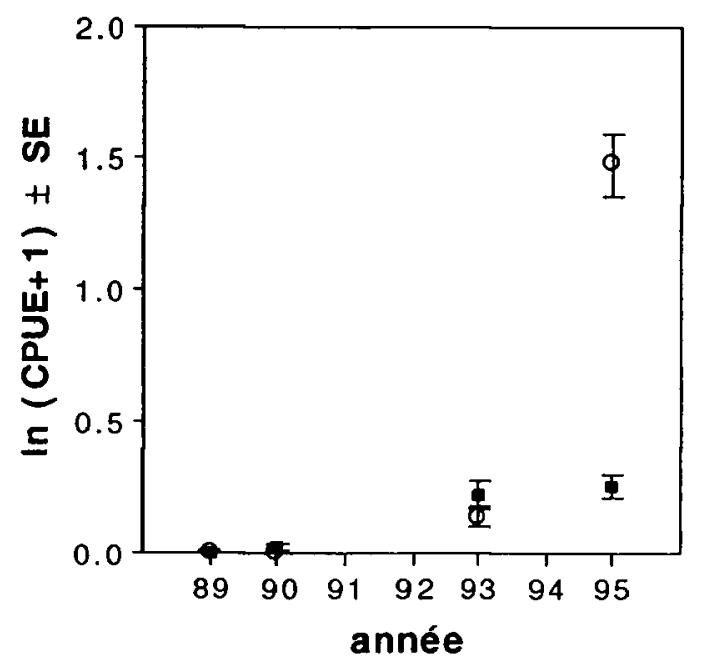

- Pseudorasbora parva

- Carassius auratus gibelio

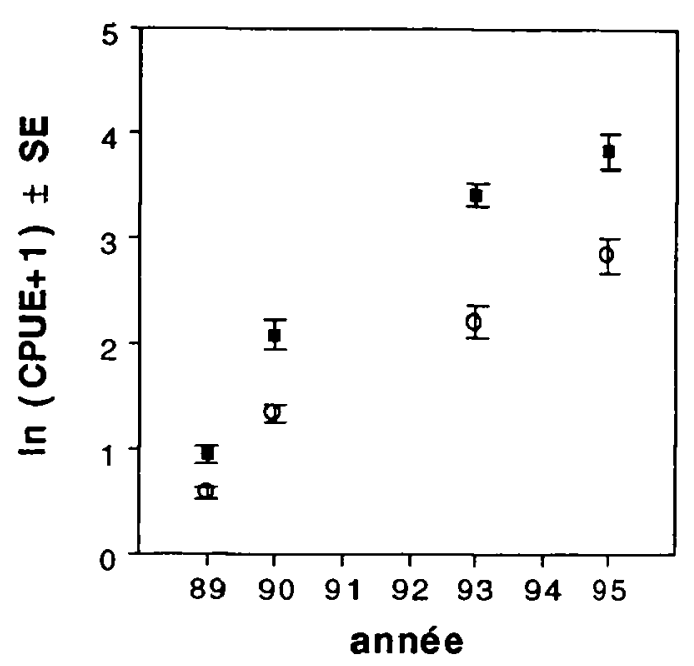

- espèces autochtones

- espèces exotiques

Figure 2

Evolution des captures d'espèces autochtones et exotiques de poissons dans le canal de drainage du Fumemorte entre 1989 et 1995. Les captures de deux espèces exotiques, Pseudorasbora parva et Carassius auratus, sont illustrées en exemple.

Figure 2

Changes in the catches of native and exotic fish species in the Fumemorte drainage canal between 1989 and 1995. The catches of two introduced species, Pseudorasbora parva and Carassius auratus, are shown as examples.

Les captures totales de poissons dans les ganguis ont augmenté dans le canal du Fumemorte entre 1989 et 1995 (Fig. 2). Cet accroissement s'explique en partie par la période de sécheresse qui a marqué les années 1989 et 1990, durant lesquelles les captures sont restées faibles. Les captures d'espèces exotiques (comme Carassius et Pseudorasbora) et d'espèces autochtones augmentent parallèlement entre 1989 et 1995 (Fig. 2). La proportion (\% en nombre d'individus) des espèces exotiques dans les captures semble s'accroître légèrement : $28 \%$ en $1989,27 \%$ en $1990,33 \%$ en 1993 et $33 \%$ en 1995.

\section{Partage des ressources alimentaires dans le canal du Fumemorte}

Si l'on considère l'ensemble des espèces, dans le plan F1-F2 de l'AFC qui représente $58 \%$ des variations entre espèces, on distingue trois groupes caractérisés par des régimes alimentaires typés : l'ablette (la seule qui se nourrit fréquemment d'insectes aériens), un groupe formé du brochet, du silure et du sandre (piscivores), et un troisième groupe comprenant toutes les autres espèces (omnivores). 


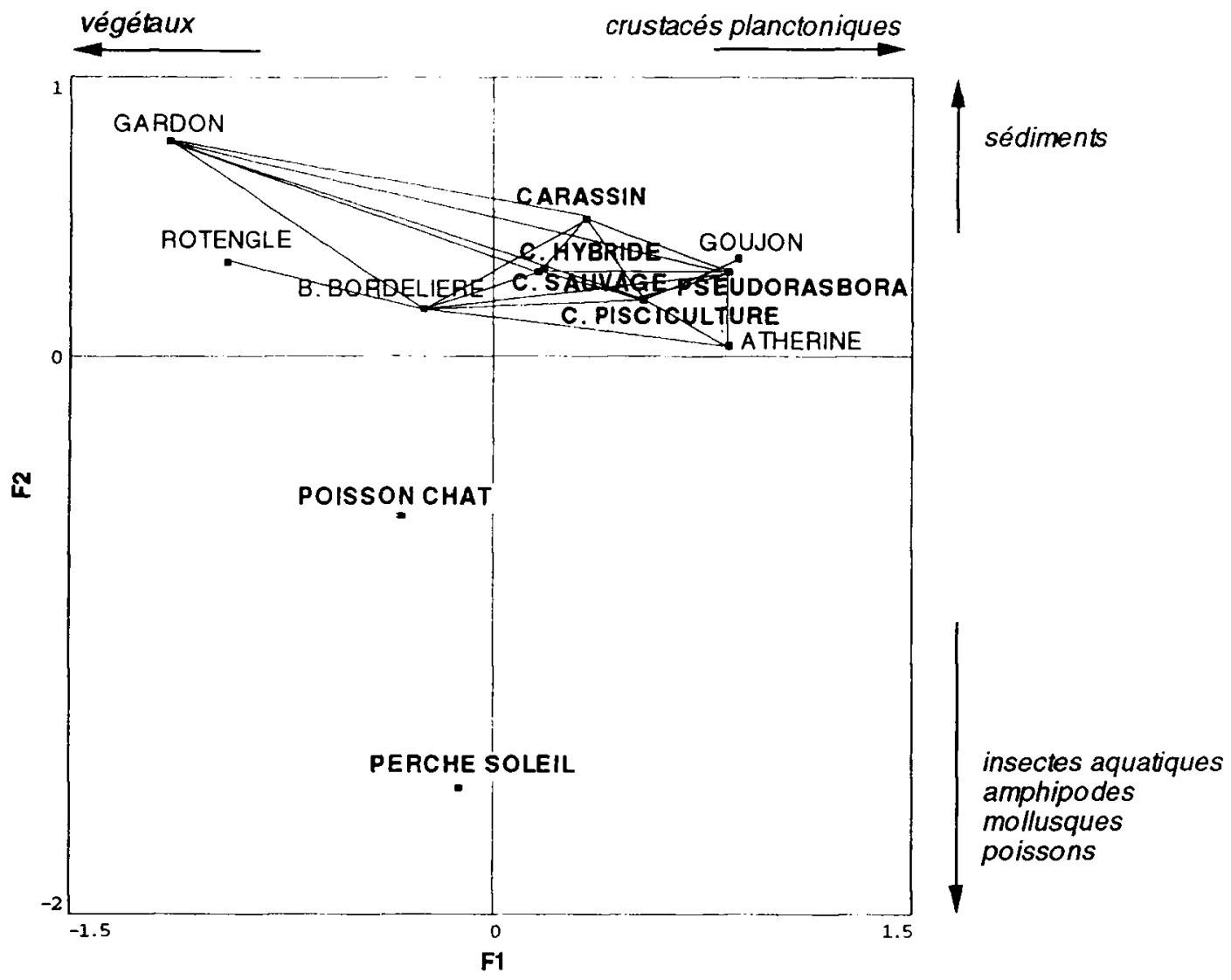

Figure 3

Plan F1 x F2 (68\% des variations totales) de l'analyse factorielle des correspondances réalisée à partir des fréquences d'occurrence de 16 catégories alimentaires dans les contenus stomacaux de 12 espèces. Les espèces introduites sont notées en gras. Les traits reliant deux espèces entre elles indiquent un coefficient de corrélation de rang de Spearman significatif $(p<0,05)$.

\section{Figure 3}

F1 x F2 map (68\% of total variations) of a correspondence analysis performed on frequency occurrence of 16 food categories in the stomach contents of 12 species. Introduced species are written in bold. Lines connecting two species indicate a significant Spearman rank correlation coefficient $(p<0.05)$.

L'ablette et les trois espèces piscivores ont été ôtées dans une deuxième AFC (Fig. 3). Le plan F1 x F2 représente $68 \%$ des variations entre espèces. On distingue un groupe d'espèces se nourrissant essentiellement de crustacés planctoniques (mais également de larves d'insectes et de végétaux macrophytes), parmi lesquelles les cinq Cyprinidés introduits: Pseudorasbora, Carassius et les trois variétés de carpes (Fig. 3). II y a chevauchement des régimes alimentaires entre ces cinq omnivores et quelques espèces autochtones, en particulier la brème bordelière (Spearman ; 0,529< $r<0,768,14$ ddl) et le gardon (Spearman ; $0,499<r<0,648,14 \mathrm{ddl})$. Un deuxième groupe comprend les omnivores à tendance herbivore, comme le gardon et le rotengle (Fig. 3). Le troisième groupe ne comporte que le poisson-chat et la perche-soleil, au spectre alimentaire très large et diversifié, dont les niches alimentaires ne présentent de chevauchement avec aucune autre espèce (Spearman ; 0,005<r<0,467, 14 ddl). 


\section{Stratégies démographiques de Pseudorasbora parva dans le canal du Fumemorte}

C'est un Cyprinidé asiatique dont la distribution originale couvre le Japon, la Corée, la Chine et le bassin du fleuve Amour. Introduit accidentellement dans des bassins du Delta du Danube en 1961, il a depuis établi une distribution pan-danubienne (BIANCO, 1988). II est également présent dans de nombreux autres pays, comme la France (ALLARDI et CHANCEREL, 1988) et la Grèce (ROSECCHI et al., 1993). En 1993, il a été signalé pour la première fois dans le canal du Fumemorte (ROSECCHI et al., 1993).

Dans le Fumemorte, où la croissance reprend dès le mois de mars, toutes les écailles présentent un accroissement marginal. Le maximum d'anneaux d'arrêt de croissance observés est 2 , la classe $1+$ représentant environ $70 \%$ des effectifs. Cette espèce aurait donc une faible longévité. Les tailles moyennes des deux classes d'âge, déterminées par la lecture d'âge, sont de $55,6 \mathrm{~mm}(n=48$, écart-type $=8,1)$ et de $75,7 \mathrm{~mm}(n=21$, écart-type $=6,3)$ respectivement.

Tous les individus sont matures dès la première saison de ponte qui suit leur naissance. La saison de ponte dans ce milieu dure d'avril à juillet. Chez les mâles, le rapport gonadosomatique moyen montre un plateau en avril, mai et juin ; il reste stable $(1,65 \%-2 \%)$ sur ces trois mois (ANOVA, $F=7,077 ; 6$ et $35 \mathrm{ddl}, \mathrm{p}<0,001)$. Chez les femelles, le pic en mai $(27,87 \%)$ est significativement différent des autres mois (ANOVA, $F=56,591 ; 6$ et $72 \mathrm{ddl}, \mathrm{p}<0,001$ ).

La distribution de fréquences de tailles des ovocytes dans la gonade est polymodale durant toute la saison de ponte, ce qui laisse supposer une ponte multiple. Le diamètre moyen des ovules prêts à pondre est de $0,117 \mathrm{~mm}(n=11$, écart-type 0,006$)$ et ne varie pas avec la taille de la femelle $\left(r^{2}=0,055, n=11, p>0,05\right)$.

La fécondité (FEC) est corrélée au poids et à la longueur des femelles, après transformation logarithmique :

$$
\begin{aligned}
& \operatorname{Ln} \text { FEC }=2,256 \text { Ln LF - 2, } 946\left(n=32, r^{2}=0,707, p<0,001\right) \\
& \text { Ln FEC }=0,785 \text { Ln PE }+5,589\left(n=32, r^{2}=0,700, p<0,001\right)
\end{aligned}
$$

\section{Comparaison des paramètres démographiques de deux populations de Pseudorasbora parva}

Nous n'avons pas pu comparer les traits d'histoire de vie de Pseudorasbora dans le Fumemorte à ceux observés dans sa zone de distribution naturelle, les données portant sur la biologie de cette espèce dans son milieu naturel étant rares et éparses. Nous avons comparé certains paramètres démographiques de Pseudorasbora dans le Fumemorte, à ceux observés dans le lac Mikri Prespa en Grèce (ROSECCHI et al., 1993). Le pic (en mai) du rapport gonadosomatique (RGS) moyen chez les femelles du Fumemorte $(27,87 \%)$ est supérieur à celui des femelles de Prespa (13,68\%) (ANOVA, $F=103,79 ; 1$ et 43 ddl, $p<0,001$ ). La condition moyenne en mai dans le Fumemorte (1,609 chez les femelles ; 1,587 chez les mâles) est supérieure à celle observée dans le lac Prespa (1,256 chez les femelles ; 1,264 chez les mâles). Cette différence est significative pour les femelles (ANOVA, $F=80,74 ; 1$ et 43 ddl, $p<0,001$ ) et les mâles (ANOVA, $F=22,15 ; 1$ et 20 ddl, $p<0,001$ ). Enfin, la fécondité relative à la longueur est significativement plus élevée dans le Fumemorte (ANCOVA, $F=36,676, p<0,001$ ).

L'alimentation de Pseudorasbora dans le Fumemorte diffère significativement de celle dans le lac Prespa (Spearman, $r=0,426 ; 11 \mathrm{ddl}$ ). Les crustacés Amphipodes, Ostracodes et Décapodes sont notamment plus abondants dans les contenus stomacaux des Pseudorasbora de Prespa. Ceci démontre la grande euryphagie de cette espèce, qui lui permet l'exploitation des ressources de milieux très différents.

\section{Hybridation entre carpes sauvage et domestique}

Certaines introductions ont eu lieu il y a si longtemps que les espèces exotiques concernées sont souvent considérées comme indigènes, c'est le cas de la carpe, introduite au Moyen-Age et probablement même déjà à l'époque romaine (BALON, 1995). 

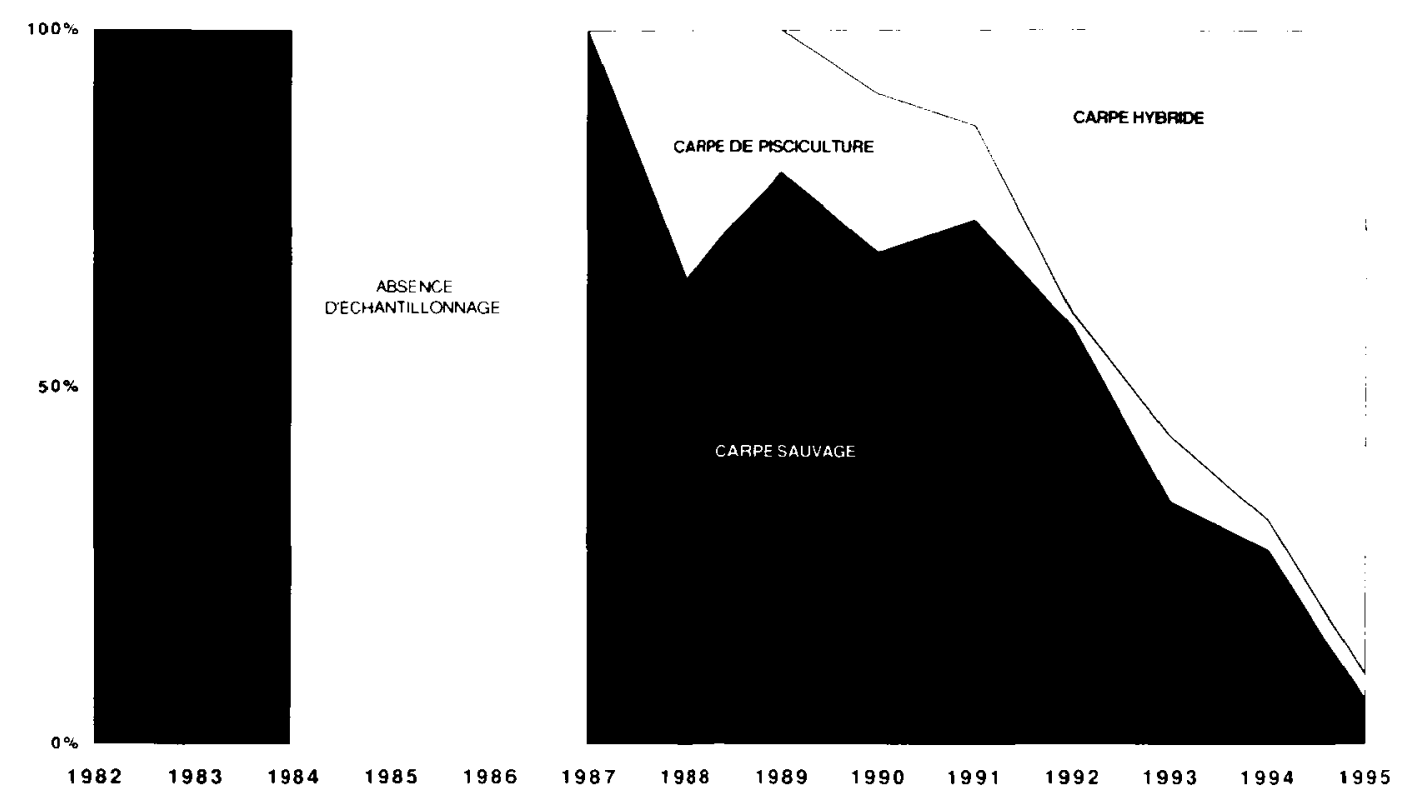

\section{Figure 4}

Evolution temporelle des abondances relatives de trois formes de carpes (sauvage, de pisciculture, hybride) dans les captures aux filets maillants, dans le canal de drainage du Fumemorte.

\section{Figure 4}

Temporal changes in the relative abundances of three carp forms (common, mirror, hybrid) caught in gill nets, in the Fumemorte drainage canal.

En 1982, seules les carpes communes étaient présentes dans le Fumemorte (Fig. 4). A partir de 1988, des carpes de pisciculture (variété miroir) furent capturées dans le canal du Fumemorte, et dès 1990 sont apparus des spécimens de carpes dont la morphologie semblait intermédiaire entre celles de la carpe commune et de la carpe miroir. Nous avons émis l'hypothèse d'une hybridation entre la forme dite sauvage, introduite anciennement, et la forme domestique, récemment introduite. Depuis 1990, la proportion de formes hybrides n'a cessé d'augmenter dans les captures et, à l'heure actuelle, les formes sauvages sont devenues très rares dans nos prises (Fig. 4).

Les formes sauvage et de pisciculture se distinguent par leur nombre d'écailles. Dans notre échantillon, le nombre d'écailles sur la ligne latérale varie entre 34 et 39 pour la carpe sauvage $(N=28)$, et entre 0 et 5 pour la carpe de pisciculture $(N=7)$. Les individus supposés hybrides ont des nombres d'écailles semblables aux carpes sauvages, entre 35 et $39(N=22)$.

Une série de 17 variables morpho-méristiques ont été mesurées sur notre échantillon de carpes et l'analyse discriminante a été appliquée sans tenir compte du nombre d'écailles. On observe que les carpes supposées hybrides forment un continuum entre les carpes sauvage et de pisciculture, du point de vue morpho-méristique (Fig. 5).

Ces différences morphologiques ont été confortées par une comparaison des relations taille-poids des trois formes, la carpe miroir ayant la plus forte croissance pondérale et la carpe hybride ayant une croissance intermédiaire entre les deux formes parentales (ANCOVA, $F=28,4$ $(2 ; 567), p<0,001)$. 


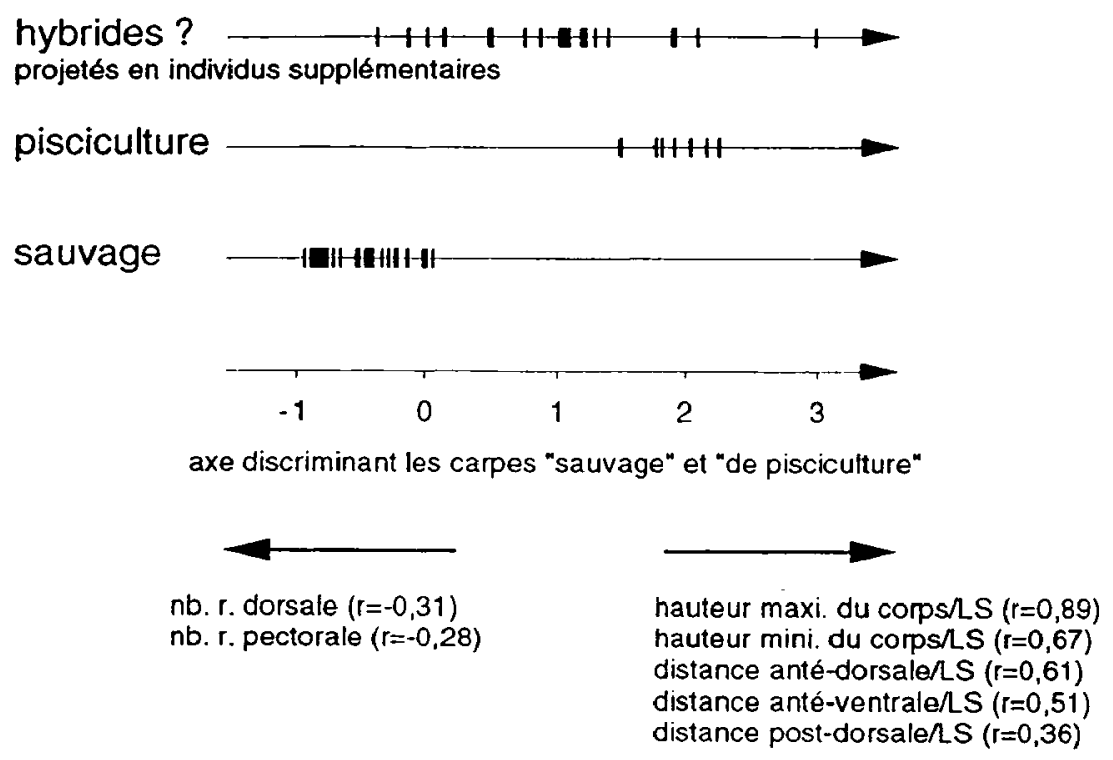

\section{Figure 5}

Analyse discriminante des carpes sauvage $(N=28)$ et de pisciculture $(N=7)$ sur 17 variables morpho-méristiques. Les variables les plus corrélées avec l'axe discriminant sont indiquées, ainsi que leur coefficient de corrélation $r$ (nb. $r$. : nombre de rayons à la nageoire ; LS : longueur standard).

\section{Figure 5}

Discriminant analysis of common carp $(\mathbf{N}=28)$ and mirror carp $(\mathbf{N}=7)$ using 17 morpho-meristic variables. The variables most correlated to the discriminant axis are indicated, along with their correlation coefficient $r$ (nb. r. : number of rays on the fin ; LS : standard length).

\section{DISCUSSION}

Resté stable entre 1957 et 1980, le nombre d'espèces introduites n'a depuis cessé d'augmenter en Camargue, en particulier dans le canal du Fumemorte, jusqu'à représenter 33\% des effectifs et 11 espèces sur un total de 36 capturées dans le canal du Fumemorte. La proportion des effectifs est un meilleur indicateur de l'importance quantitative des espèces introduites que ne l'est la proportion du nombre d'espèces, bien que cette dernière soit plus souvent considérée dans la littérature. A titre de comparaison, dans un secteur du fleuve Rhône, situé à environ $30 \mathrm{~km}$ au nord du canal du Fumemorte, les espèces introduites représentent $14 \%$ des effectifs échantillonnés par pêche électrique de 1989 à 1991, et 8 espèces sur un total de 39 (POIZAT, 1993). Pour cette période, la proportion des effectifs d'espèces introduites en Camargue $(27 \%)$ était supérieure à celle observée dans le Rhône, sous réserve que les différentes méthodes d'échantillonnage n'induisent pas de biais majeur.

Parmi les espèces exotiques recensées en Camargue, certaines font partie des 25 espèces globalement considérées comme nuisibles à l'échelle mondiale (WELCOMME, 1992): Pseudorasbora parva, Lepomis gibbosus et /ctalurus melas. Pourtant, malgré l'augmentation d'espèces exotiques, on ne constate pas de diminution dans les populations d'espèces autochtones dans le canal du Fumemorte, après sept années d'échantillonnage. Seule la population de perche commune, Perca fluviatilis, de l'étang du Vaccarès semble avoir brutalement décliné à la suite de l'implantation du sandre, Stizostedion lucioperca, il y a environ quarante ans (KIENER, 1968). Les impacts liés à l'introduction de grands prédateurs sont souvent plus probants, car plus spectaculaires (ROSS, 1991 ; WELCOMME, 1992 ; WINFIELD, 1992 ; LODGE, 1993). Dans le canal du Fumemorte et le Vaccarès, à l'exception du brochet, les grands prédateurs piscivores sont tous introduits (sandre, black-bass et silure). 
L'utilisation de critères morpho-méristiques pour identifier des hybrides et leurs espèces parentales est certes moins pertinente que l'étude enzymatique, mais cette dernière se révélerait délicate étant donné que cette espèce est domestiquée et manipulée génétiquement depuis des siècles (P. BERREBI, comm. pers.). Par ailleurs, l'utilisation de critères morphologiques a plutôt tendance à sous-estimer l'importance numérique de l'hybridation (FERGUSON, 1989), ce qui rend l'analyse plutôt conservatrice. L'analyse morpho-méristique confirme l'hypothèse d'hybridation dans la mesure où les hybrides ont des caractéristiques intermédiaires entre les deux formes parentales. Cependant, la grande variabilité des hybrides formant un continuum entre la forme sauvage et la forme de pisciculture suggère l'existence d'une introgression, c'est-à-dire de reproduction entre hybrides et formes parentales.

Par ailleurs, nos résultats montrent clairement que la carpe sauvage est en train de diminuer dans le canal du Fumemorte, au profit des hybrides (Fig. 4). La vitesse d'augmentation relative des hybrides par rapport aux formes sauvages et domestiques renforce encore l'hypothèse d'introgression. Cette hybridation pourrait entraîner une perte de biodiversité génétique par disparition de la forme parentale sauvage. Une diminution similaire de carpe sauvage est observee en Australie, au profit des hybrides avec une carpe miroir (ARTHINGTON, 1991).

Les chevauchements des niches alimentaires des espèces introduites et des espèces indigènes indiquent que, si les ressources disponibles étaient en quantité limitante, il pourrait y avoir compétition. Cela pourrait arriver si la salinité de l'étang du Vaccarès augmentait à nouveau, comme cela s'est produit en 1981, conduisant à de très fortes densités de population des espèces dulçaquicoles dans le canal du Fumemorte (CRIVELLI, données non publiées).

Les impacts potentiels des espèces introduites en Camargue ont trait à la compétition trophique et spatiale ( $P$ seudorasbora, carpes, carassin doré), la prédation sur les espèces de poissons autochtones (par le silure, black-bass, sandre) et sur les autres groupes animaux et végétaux, l'hybridation (carpe miroir) ou encore la dégradation de l'habitat (Procambarus clarkii). De façon générale, les rôles de la prédation et de la compétition, souvent invoqués pour expliquer le déclin d'espèces autochtones ou leur élimination par une espèce introduite, sont rarement démontrés de façon concluante, étant donné les difficultés méthodologiques rencontrées (MOYLE, 1976 ; TAYLOR et al., 1984 ; ROSS, 1991). La prédiction d'interactions trophiques entre espèces introduites et autochtones se complique encore lorsque les spectres alimentaires sont vastes et lorsqu'une espece est opportuniste, comme c'est le cas pour Pseudorasbora. Dans le canal du Fumemorte, il est difficile de prédire pour le moment quel va être l'impact néfaste de cet enrichissement sur les populations autochtones.

Le succès d'une espèce invasive dépend à la fois de ses propres caractéristiques et de celles de l'écosystème dans lequel elle va être introduite (TAYLOR et al., 1984). Etant donné la diversité écologique et biologique des espèces invasives et des espèces introduites qui se sont établies avec succès, la recherche de caractères universels parait impossible (LODGE, 1993 ; MILLS et al., 1994). En outre, un poisson peut changer ses stratégies démographiques en l'espace d'une génération, en réponse à l'environnement (BRUTON, 1986). Cependant, certains traits d'histoire de vie prédisposent à l'invasion (TAYLOR et al., 1984 ; RUESINK et al., 1995). On retrouve plusieurs de ces traits chez Pseudorasbora parva : temps de génération court, fort investissement dans la reproduction, grande tolérance aux variations climatiques et aux perturbations humaines, exigences très flexibles en ce qui concerne l'habitat, polyphagie. Ces mêmes stratégies sont d'ailleurs observées chez de nombreuses espèces en Camargue, comme la perche-soleil, et pourraient être une adaptation à un environnement imprévisible dans lequel la probabilité de survie est faible en raison du climat méditerranéen (CRIVELLI et MESTRE, 1988). En considérant les guildes reproductives de BALON (1975), plusieurs espèces introduites en Camargue présentent un mode de reproduction original, comme la construction de nids et la garde de la ponte (poisson-chat, perche-soleil, black-bass, sandre, silure, Pseudorasbora). Ces stratégies reproductives, en favorisant la survie de la progéniture, vont permettre une augmentation plus rapide de la population. Un autre avantage lié à la construction d'un nid est de pouvoir survivre dans des eaux de moindre qualité, les parents 
nettoyant les oeufs. Ceci est à prendre en considération dans un canal de drainage, les fonds vaseux du Fumemorte n'ayant pas permis le succès reproductif du sandre dont les oeufs doivent être bien oxygénés. Chez la gambusie vivipare, la femelle de même que sa progéniture se reproduisent plusieurs fois au cours d'une seule saison de ponte, de grandes populations peuvent donc être établies même si la mortalité est élevée. Enfin la gynogenèse, chez le carassin, Carassius auratus gibelio, permet une augmentation de la fécondité totale de la population, plus de $90 \%$ des individus étant des femelles.

\section{CONCLUSION}

II semble nécessaire d'améliorer les méthodes permettant d'étudier les interactions entre espèces introduites et autochtones. L'un des moyens consiste à comparer simultanément des populations d'une même espèce autochtone dans deux zones d'études comparables et suffisamment proches géographiquement, l'une en présence d'une espèce exotique, l'autre en l'absence d'espèce exotique. D'autres études intègrent les connaissances sur l'écologie des espèces introduites dans leur aire de répartition naturelle (TOWNSEND et WINTERBOURN, 1992), qui peuvent permettre l'élaboration de modèles prédictifs (COATES et ULAIWI, 1995). Dans notre étude, nous avons choisi de comparer le devenir d'une espèce exotique, Pseudorasbora parva, introduite dans deux milieux aux conditions climatiques et environnementales très différentes : le lac Mikri Prespa en Grèce et le canal du Fumemorte. De cette comparaison, il ressort d'une part que la plasticité phénotypique de Pseudorasbora prédispose cette espèce à coloniser de nouveaux habitats, d'autre part que cette espèce aurait trouvé en Camargue des conditions optimales pour sa prolifération. Sa présence et sa reproduction dans le Vaccarès depuis 1993 semblent soutenir cette hypothèse. II reste néanmoins que seules des études à long terme nous permettront réellement d'évaluer le devenir des espèces introduites et leurs impacts sur les populations autochtones de poissons.

\section{BIBLIOGRAPHIE}

ALLARDI J., CHANCEREL F., 1988. Note ichtyologique sur la présence en France de Pseudorasbora parva (Schlegel, 1842). Bull. Fr. Pêche Piscic., 308, 35-37.

ARTHINGTON A.H., 1991. Ecological and genetic impacts of introduced and translocated freshwater fishes in Australia. Can. J. Fish. Aquat. Sci., 48 (suppl.1), 33-43.

BAIN M., 1993. Assessing impacts of introduced aquatic species : grass carp in large systems. Environ. Manage., 17 (2), 211-224.

BALON E.K., 1975. Reproductive guilds of fishes : a proposal and definition. J. Fish. Res. Board Can., 32 (6), 821-864.

BALON E.K., 1995. Origin and domestication of the wild carp, Cyprinus carpio : from Roman gourmets to the swimming flowers. Aquaculture, 129, 3-48.

BIANCO P.G., 1988. Occurrence of the Asiatic gobionid Pseudorasbora parva (Temminck and Schlegel) in south-eastern Europe. J. Fish Biol., 32, 973-974.

BRUTON M.N., 1986. Life history styles of invasive fishes in southern Africa. In MACDONALD I.A.W., KRUGER F.J., FERRAR A.A., Ecology and management of biological invasions in South Africa, 201-208, Oxford University Press, Londres.

CHESSEL D., DOLEDEC S., 1994. Programmathèque ADE. Analyses multivariées et expression graphique des données environnementales (version 3.7). 9 volumes, Université Lyon 1 , Villeurbanne, $871 \mathrm{p}$.

CHIMITZ P., 1947. Note sur l'acclimatation de Gambusia holbrooki dans les étangs des Landes. Bull. Fr. Piscic., 147, 79-82. 
COATES D., ULAIWI W.K., 1995. A simple model for predicting ecological impacts of introduced aquatic organisms : a case study of common carp, Cyprinus carpio L., in the Sepik-Ramu river basin, Papua New Guinea. Fisheries Management and Ecology, 2, 227-242.

COURTENAY Jr. W.R., 1989. Fish introductions and translocations, and their impacts in Australia. Bureau of Rural Resources Proceedings, 8, 171-179.

CRIVELLI A.J., 1981. Les peuplements de poissons de Camargue. Rev. Ecol. Terre Vie, 35, 617-671.

CRIVELLI A.J., 1995. Are introduced species a threat to endemic freshwater fishes in the northern Mediterranean region ? Biol. Conserv., 72, 311-319.

CRIVELLI A.J., MESTRE D., 1988. Life history traits of pumpkinseed, Lepomis gibbosus, introduced into the Camargue, a Mediterranean wetland. Arch. Hydrobiol., 111 (3), 449-466.

FERGUSON M.M., 1989. The genetic impact of introduced fishes on native species. Can. J. Zool., 68, 1053-1057.

KIENER A., 1968. L'évolution des populations de sandre dans deux étangs méditerranéens. Terre Vie, 4, 470-491.

LÉVÊQUE R., 1957. Notes sur la faune ichtyologique de Camargue. Terre Vie, 104, 231-240.

LODGE D., 1993. Biological invasions : lessons for ecology. Tree, 8 (4), 133-137.

MILLS E.L., LEACH J.H., CARLTON J.T., SECOR C.L., 1994. Exotic species and the integrity of the Great Lakes. Bioscience, 44 (10), 666-676.

MOYLE P.B., 1976. Fish introduction in California : history and impact on native fish. Biol. Conser., 9, 101-118.

PETIT G., 1953. Introduction à l'étude écologique des étangs méditerranéens. Vie Milieu, 4, 576-604.

POIZAT G., 1993. Echelle d'observation et variabilité des abondances de juvéniles de poissons dans un secteur aval du Rhône. Thèse de Doctorat, Université Claude Bernard Lyon I, $217 \mathrm{p}$.

ROQUEPLO C., LAURENT P.J., NEVEU A., 1995. Procambarus clarkii Girard, synthèse sur les problèmes posés par cette espèce et sur les essais pour contrôler ses populations. Première partie. L'astaciculteur de France, 44, 2-14.

ROSECCHI E., CRIVELLI A.J., CATSADORAKIS G., 1993. The establishment and impact of Pseudorasbora parva, an exotic fish species introduced into Lake Mikri Prespa (north-western Greece). Aquatic Conservation: Marine and Freshwater Ecosystems, 3, 223-231.

ROSS S.T., 1991. Mechanisms structuring stream fish assemblages : are there lessons from introduced species ? Environ. Biol. Fish, 30, 359-368.

RUESINK J.L., PARKER I.M., GROOM M.J., KAREIVA P.M., 1995. Reducing the risks of nonindigenous species introductions. Bioscience, 45 (7), 465-477.

TAYLOR J.N., COURTENAY Jr. W.R., MCCANN J.A., 1984. Known impacts of exotic fishes in the continental United States in COURTENAY Jr. W.R., STAUFFER Jr. J.R., Distribution, Biology and Management of exotic fishes, 323-373, John Hopkins University Press, Baltimore.

TOWNSEND C.R., WINTERBOURN M.J., 1992. Assessment of the environmental risk posed by an exotic fish : the proposed introduction of channel catfish (Ictalurus punctatus) to New Zealand. Conserv. Biol., 6 (2), 273-282.

WELCOMME R.L., 1992. A history of international introductions of inland aquatic species. ICES mar. Sci. Symp.,194, 3-14.

WINFIELD I.J., 1992. Threats to the lake fish communities of the U.K. arising from eutrophication and species introductions. Neth. J. Zool., 42 (2-3), 233-242. 